\title{
Bilateral transient olfactory bulb edema during COVID-19-related anosmia
}

Thomas Laurendon, MD, Thomas Radulesco, MD, PhD, Justine Mugnier, MD, Mélanie Gérault, PhD, Christophe Chagnaud, MD, PhD, Ahmed-Ali El Ahmadi, MD, and Arthur Varoquaux, MD, PhD

Neurology ${ }^{\circledR}$ 2020;95:224-225. doi:10.1212/WNL.0000000000009850

Figure Transient olfactory bulb edema
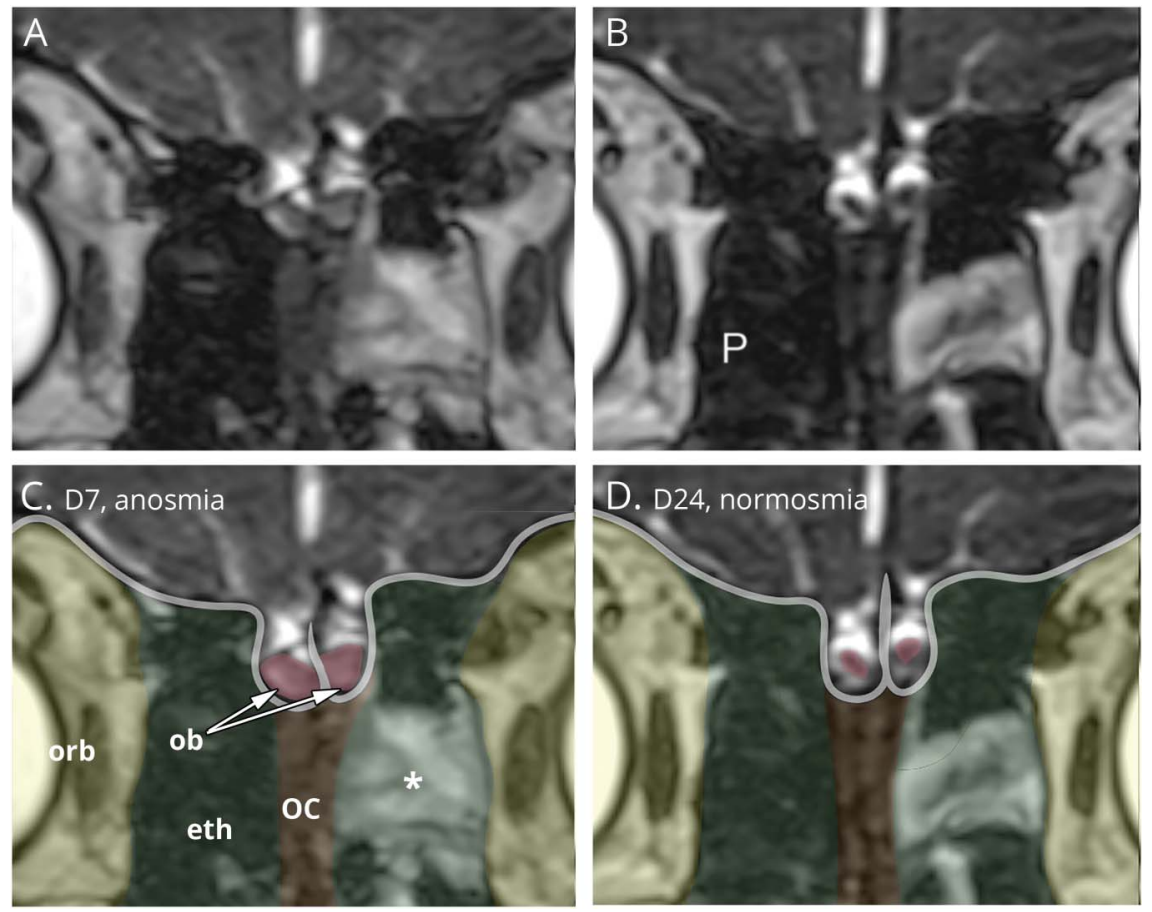

Coronal 3D constructive interference in steady-state T2-weighted imaging (1.5T) during anosmia (day 7; A, C) compared to recovery (day 24; B, D). MRI shows olfactory bulb (ob; pink) transient volume and signal increase, olfactory cleft edema (OC; brown), and focal left ethmoid (eth; green) sinusitis ( $\left.{ }^{*}\right)$, and normal cranial fossa (gray line) and orbit (orb; yellow).

An asymptomatic 27-year-old man was diagnosed with coronavirus disease 2019 (COVID-19) by occupational medicine after contagion (reverse transcription polymerase chain reaction $[R T-P C R])$. Four days after the diagnosis, he experienced complete anosmia and dysgeusia. ${ }^{1}$ On day 7, $1.5 \mathrm{~T}$ MRI showed signs of bilateral olfactory bulb edema on $3 \mathrm{D}$ constructive interference in steady state T2-weighted imaging, demonstrated by severe enlargement ${ }^{2}$ (left: $73 \mathrm{~mm}^{3}$, right: $64 \mathrm{~mm}^{3}$ ) and an abnormally high signal intensity (figure). Olfactory clefts showed mild edema. The olfactory pathways, including the cortical projections (fluidattenuated inversion recovery and diffusion-weighted imaging not shown), were normal. Sensory recovery and negative RT-PCR (positive on days 1, 2, and 10) appeared on day 14.

\section{Correspondence}

Prof. Varoquaux

arthur.varoquaux@ap-hm.fr or Arthur@Varoquaux.com

\section{MORE ONLINE}

\section{COVID-19 Resources}

For the latest articles, invited commentaries, and blogs from physicians around the world NPub.org/COVID19

From the Departments of Medical Imaging (T.L., J.M., C.C., A.-A.E.A., A.V.) and ENT Surgery (T.R.), Conception University Hospital, and Center for Magnetic Resonance in Biology and Medicine (C.C., A.V.), UMR 7339, La Timone University Hospital, Aix-Marseille University, France; and Department of Earth, Atmospheric and Planetary Sciences (M.G.), Massachusetts Institute of Technology, Cambridge.

Go to Neurology.org/N for full disclosures. Funding information and disclosures deemed relevant by the authors, if any, are provided at the end of the article. 
MRI on day 24 confirmed the normalization of olfactory bulb signal and volumes (left: $22 \mathrm{~mm}^{3}$, right: $17 \mathrm{~mm}^{3}$ ).

\section{Study funding}

No targeted funding reported.

\section{Disclosure}

The authors report no disclosures relevant to the manuscript. Go to Neurology.org/N for full disclosures.

\section{Appendix Authors}

\begin{tabular}{|c|c|c|}
\hline Name & Location & Contribution \\
\hline $\begin{array}{l}\text { Thomas } \\
\text { Laurendon, } \\
\text { MD }\end{array}$ & $\begin{array}{l}\text { Department of Medical } \\
\text { Imaging, Conception } \\
\text { University Hospital, Aix- } \\
\text { Marseille University, } \\
\text { Marseille, France }\end{array}$ & $\begin{array}{l}\text { Traced the patient's entire } \\
\text { history, including biological } \\
\text { history and management, } \\
\text { wrote the manuscript }\end{array}$ \\
\hline $\begin{array}{l}\text { Thomas } \\
\text { Radulesco, } \\
\text { MD, PhD }\end{array}$ & $\begin{array}{l}\text { Department of ENT } \\
\text { surgery, Conception } \\
\text { University Hospital, Aix- } \\
\text { Marseille University, } \\
\text { Marseille, France }\end{array}$ & $\begin{array}{l}\text { Examined the patient and } \\
\text { performed sensory testing, } \\
\text { corrected the manuscript }\end{array}$ \\
\hline $\begin{array}{l}\text { Justine } \\
\text { Mugnier, } \\
\text { MD }\end{array}$ & $\begin{array}{l}\text { Department of Medical } \\
\text { Imaging, Conception } \\
\text { University Hospital, Aix- } \\
\text { Marseille University, } \\
\text { Marseille, France }\end{array}$ & $\begin{array}{l}\text { Performed the } \\
\text { bibliography and image } \\
\text { analysis (volume and signal } \\
\text { intensities), corrected the } \\
\text { manuscript }\end{array}$ \\
\hline $\begin{array}{l}\text { Mélanie } \\
\text { Gérault, } \\
\text { PhD }\end{array}$ & $\begin{array}{l}\text { Department of Earth, } \\
\text { Atmospheric and Planetary } \\
\text { Sciences, Massachusetts } \\
\text { Institute of Technology, } \\
\text { Cambridge }\end{array}$ & $\begin{array}{l}\text { Supervised manuscript } \\
\text { corrections and provided } \\
\text { substantial mathematical } \\
\text { help in checking the } \\
\text { volume calculations by } \\
\text { triple checking with Horos } \\
\text { program, oro.dicom under } \\
\text { R statistics, and } \\
\text { geometrical length model }\end{array}$ \\
\hline
\end{tabular}

Appendix (continued)

\begin{tabular}{|c|c|c|}
\hline Name & Location & Contribution \\
\hline $\begin{array}{l}\text { Christophe } \\
\text { Chagnaud, } \\
\text { MD, PhD }\end{array}$ & $\begin{array}{l}\text { Department of Medical } \\
\text { Imaging, Conception } \\
\text { University Hospital, Aix- } \\
\text { Marseille University; } \\
\text { Center for Magnetic } \\
\text { Resonance in Biology and } \\
\text { Medicine, La Timone } \\
\text { University Hospital, Aix- } \\
\text { Marseille University, } \\
\text { Marseille, France }\end{array}$ & $\begin{array}{l}\text { Department Chief; took } \\
\text { part in ethical } \\
\text { considerations, corrected } \\
\text { the manuscript }\end{array}$ \\
\hline $\begin{array}{l}\text { Ahmed-Ali } \\
\text { El Ahmadi, } \\
\text { MD }\end{array}$ & $\begin{array}{l}\text { Department of Medical } \\
\text { Imaging, Conception } \\
\text { University Hospital, Aix- } \\
\text { Marseille University, } \\
\text { Marseille, France }\end{array}$ & $\begin{array}{l}\text { Responsible for image } \\
\text { acquisitions, editorial, } \\
\text { manuscript correction, } \\
\text { took part in the submission } \\
\text { process }\end{array}$ \\
\hline $\begin{array}{l}\text { Arthur } \\
\text { Varoquaux, } \\
\text { MD, PhD }\end{array}$ & $\begin{array}{l}\text { Department of Medical } \\
\text { Imaging, Conception } \\
\text { University Hospital, Aix- } \\
\text { Marseille University; } \\
\text { Center for Magnetic } \\
\text { Resonance in Biology and } \\
\text { Medicine, La Timone } \\
\text { University Hospital, Aix- } \\
\text { Marseille University, } \\
\text { Marseille, France }\end{array}$ & $\begin{array}{l}\text { Supervised work, took part } \\
\text { in ethical consideration, } \\
\text { acquisition parameters, } \\
\text { manuscript correction and } \\
\text { submission }\end{array}$ \\
\hline
\end{tabular}

\section{References}

1. Lechien JR, Chiesa-Estomba CM, De Siati DR, et al. Olfactory and gustatory dysfunctions as a clinical presentation of mild-to-moderate forms of the coronavirus disease (COVID-19): a multicenter European study. Eur Arch Otorhinolaryngol Epub 2020 Apr 6. Available at: link.springer.com/10.1007/s00405-02005965-1.

2. Abolmaali ND, Hietschold V, Vogl TJ, Hüttenbrink KB, Hummel T. MR evaluation in patients with isolated anosmia since birth or early childhood. Am J Neuroradiol 2002; 23:157-164.

\section{ค}

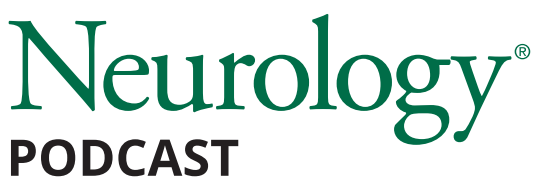

August 4, 2020

CME Opportunity: Listen to this week's Neurology Podcast and earn 0.5 AMA PRA Category 1 CME Credits ${ }^{\mathrm{TM}}$ by completing the online Podcast quiz.

\section{Wisdom of the expert crowd prediction of response for 3 neurology randomized trials (see p. 201)}

\footnotetext{
In the first segment, Dr. Jason Crowell talks with Dr. Jonathan Kimmelman about his paper on expert crowd prediction on clinical trial response. In the second part of the podcast, Dr. Andrew Southerland and Dr. J.G. Makin talk about Dr. Makin's Nature article on thought-to-text technology. The article is available online at: https://www.nature.com/articles/s41593-020-0608-8.
}

Disclosures can be found at Neurology.org. 


\section{Neurology}

\section{Bilateral transient olfactory bulb edema during COVID-19-related anosmia}

Thomas Laurendon, Thomas Radulesco, Justine Mugnier, et al.

Neurology 2020;95;224-225 Published Online before print May 22, 2020

DOI 10.1212/WNL.0000000000009850

This information is current as of May 22, 2020

\begin{tabular}{|c|c|}
\hline $\begin{array}{l}\text { Updated Information \& } \\
\text { Services }\end{array}$ & $\begin{array}{l}\text { including high resolution figures, can be found at: } \\
\text { http://n.neurology.org/content/ } 95 / 5 / 224 \text {. full }\end{array}$ \\
\hline References & $\begin{array}{l}\text { This article cites } 1 \text { articles, } 0 \text { of which you can access for free at: } \\
\text { http://n.neurology.org/content/95/5/224. full\#ref-list- } 1\end{array}$ \\
\hline Citations & $\begin{array}{l}\text { This article has been cited by } 10 \text { HighWire-hosted articles: } \\
\text { http://n.neurology.org/content/95/5/224.full\#\#otherarticles }\end{array}$ \\
\hline Subspecialty Collections & $\begin{array}{l}\text { This article, along with others on similar topics, appears in the } \\
\text { following collection(s): } \\
\text { MRI } \\
\text { http://n.neurology.org/cgi/collection/mri }\end{array}$ \\
\hline Permissions \& Licensing & $\begin{array}{l}\text { Information about reproducing this article in parts (figures,tables) or in } \\
\text { its entirety can be found online at: } \\
\text { http://www.neurology.org/about/about_the_journal\#permissions }\end{array}$ \\
\hline Reprints & $\begin{array}{l}\text { Information about ordering reprints can be found online: } \\
\text { http://n.neurology.org/subscribers/advertise }\end{array}$ \\
\hline
\end{tabular}

Neurology ${ }^{\circledR}$ is the official journal of the American Academy of Neurology. Published continuously since 1951, it is now a weekly with 48 issues per year. Copyright @ 2020 American Academy of Neurology. All rights reserved. Print ISSN: 0028-3878. Online ISSN: 1526-632X.

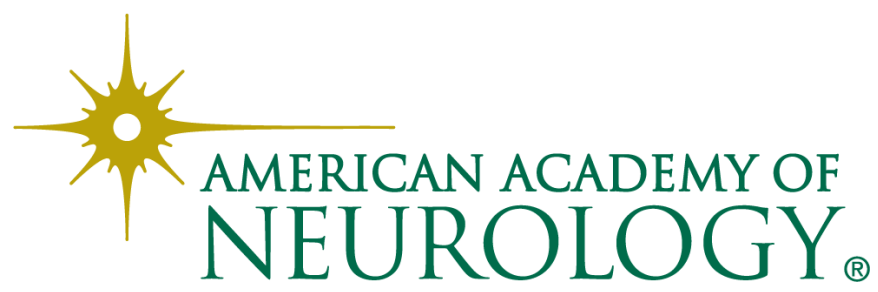

